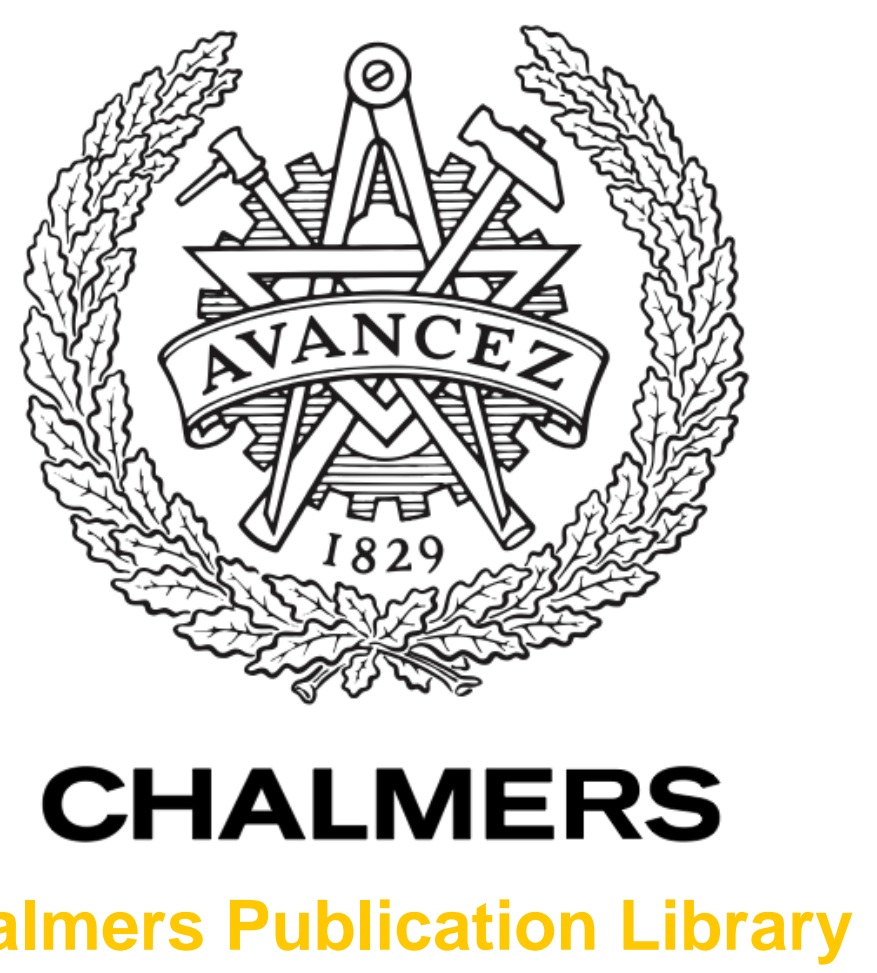

\title{
On joint energy and information transfer in relay networks with an imperfect power amplifier
}

This document has been downloaded from Chalmers Publication Library (CPL). It is the author's version of a work that was accepted for publication in:

\section{PIMRC}

Citation for the published paper:

haghifam, m. ; Makki, B. ; kenari, m. et al. (2016) "On joint energy and information transfer in relay networks with an imperfect power amplifier". PIMRC

Downloaded from: http://publications.lib.chalmers.se/publication/247957

Notice: Changes introduced as a result of publishing processes such as copy-editing and formatting may not be reflected in this document. For a definitive version of this work, please refer to the published source. Please note that access to the published version might require a subscription. 


\title{
On joint energy and information transfer in relay networks with an imperfect power amplifier
}

\author{
Mahdi Haghifam ${ }^{\star}$, Behrooz Makki ${ }^{\dagger}$, Masoumeh Nasiri-Kenari ${ }^{\star}$, Tommy Svensson ${ }^{\dagger}$ \\ ${ }^{\star}$ Electrical Engineering Department, Sharif University of Technology, Tehran, Iran \\ haghifam_mahdi@ee.sharif.edu, mnasiri@sharif.edu \\ ${ }^{\dagger}$ Department of Signals and Systems, Chalmers University of Technology, Gothenburg, Sweden \\ \{behrooz.makki, tommy.svensson\} @ chalmers.se
}

\begin{abstract}
This paper investigates the outage probability and the throughput of relay networks with joint information and energy transfer where the relay harvests energy from transmitted radio-frequency (RF) signal of the source. Considering different power consumption models, we derive the outage probability of the systems for both adaptive and non-adaptive power allocations at the relay. With a total energy consumption constraint at the source, we provide closed-form expressions for the optimal time sharing and power allocation between the source energy and information transfer signals. The analytical and simulation results demonstrate the efficiency of joint energy and information transfer systems in different conditions.
\end{abstract}

\section{INTRODUCTION}

One of the promising methods to ensure high quality of service in the wireless networks is to use many small cheap nodes that support information transfer between the terminals. These devices are usually powered by fixed but limited batteries. Thus, wireless networks may suffer from the short lifetime and require periodic battery replacement/recharging. However, the battery replacement may be infeasible in, e.g., biological or chemical environments. It has been recently proposed to use radio-frequency (RF) signals as a means of wireless energy transfer. Significant advances in the circuit design for RF energy transfer make the usage of energy transfer as a viable and practical solution for future wireless networks, e.g., [1].

The concept of using RF signal for simultaneously wireless information and energy transfer is introduced in [2]. Practical receiver design and modulation techniques for wireless information and energy transfer are studied in [3]. In [4], [5], relaying protocols with simultaneous wireless information and energy transfer are proposed. Moreover, [6] derives different power allocation strategies for energy harvesting relay networks with multiple source-destination pairs and a single energy harvesting relay. In [7], [8], the outage optimization in the energy harvesting networks are considered and analyzed. Relay networks with simultaneous wireless energy and information transfer are studied in [9], [10].

In this paper, we study the performance of relay networks with joint information and energy transfer and realistic assumptions on the power consumption of the relay. The design problem is cast in the form of minimizing the outage probability subject to a total energy consumption constraint at the source. We use time switching protocol for the relay-based data/energy transfer. We derive closed-form expressions for the optimal, in terms of energy-constrained outage probability, time sharing between the energy and information signals (Theorem 1). Also, we obtain closed-form expressions for the outage-optimized power allocation of the source (Theorem 2). Finally, we analyze the effect of adaptive power allocation by the relay on the network performance.

Compared to the literature, e.g., [4]-[11], we consider different power amplifier (PA) model, optimization criteria/metrics and problem formulation which lead to completely different analysis/conclusions. Moreover, our discussions on the optimal time sharing between energy and information signals, the optimal power allocations by the source and the impact of adaptive power allocation have not been presented before.

Our analytical results which have been confirmed by simulation indicate that the optimal time sharing, with respect to the source transmission power, has two regions. While in the first region, the optimal time sharing is independent of the source transmission power, in the second region, it is an increasing function of the source transmission power (Theorem 1 and Fig. 2). Furthermore, at high signal to noise rations (SNRs), the optimal power for energy transfer signal, in terms of outage probability, increases linearly with the total energy constraint of the source and decreases exponentially with the codeword rate (Theorem 2).

The outline of this paper is as follows. In Section II, the system model is described. In Sections III and IV, we analyze the system performance with non-adaptive and adaptive power allocations of the relay, respectively. The simulation results are given in Section $\mathrm{V}$, where we verify the analytic results. Finally, Section VI concludes the paper.

\section{SYSTEM MODEL}

We consider a relay-assisted cooperative communication setup consisting of a source, a relay and a destination. The source and the destination nodes have constant, e.g., wired, power supply. On the other hand, the relay has no fixed power supply and receives its required energy from the source wirelessly. The channel coefficients in the source-relay and the relay-destination links are denoted by $h_{\mathrm{sr}}$ and $h_{\mathrm{rd}}$, respectively. The channel coefficients remain constant during the channel coherence time and then change according to their probability 
density functions (PDFs). Also, we define the channel gains as $g_{\mathrm{sr}}=\left|h_{\mathrm{sr}}\right|^{2}$ and $g_{\mathrm{rd}}=\left|h_{\mathrm{rd}}\right|^{2}$.

Let us denote the total packet transmission length from the source to the destination by $T$. The energy transfer and data communication protocol are in three phases as follows. In the first phase, of length $(1-\theta) T, \theta \in[0,1]$, the relay harvests energy from the source transmitted energy signal. Let $P_{\mathrm{s}}^{\mathrm{e}}$ be the transmission power of the source during the energy transmission phase. Then, the baseband signal model in this period is given by

$$
y_{\mathrm{r}}^{\mathrm{e}}=\sqrt{P_{\mathrm{s}}^{\mathrm{e}}} h_{\mathrm{sr}}+z_{\mathrm{r}},
$$

where $z_{\mathrm{r}}$ is the additive white Gaussian noise. In this way, the energy harvested by the relay at the end of the energy transfer phase is given by $E_{\text {stored }}=(1-\theta) T \eta g_{\mathrm{s}, \mathrm{r}} P_{\mathrm{s}}^{\mathrm{e}}$, with $\eta$ representing the efficiency factor of the energy harvesting circuit. With no loss of generality, we set $\eta=1$. Also, we assume an ideal battery for the relay, such that no overflow occurs. Let us define $E_{\text {proc }}$ as the minimum energy required by the relay to process the source signal. Once the relay's required energy, $E_{\text {proc }}$, is supplied in Phase 1 , it sends one bit acknowledgement to the source, and the information transfer starts where the information is forwarded to the destination through the relay (the consumed energy for sending one bit feedback is included in the relay's minimum required energy). Receiving the acknowledgement from the relay, the second phase of length $\frac{\theta}{2} T$ starts where the source sends information to the relay. Let $x_{\mathrm{s}}^{\mathrm{i}} \in \mathcal{C N}(0,1)$ and $y_{\mathrm{r}}^{\mathrm{i}}$ be the source information signal and its corresponding received signal by the relay, respectively. Hence

$$
y_{\mathrm{r}}^{\mathrm{i}}=\sqrt{P_{\mathrm{s}}^{\mathrm{i}}} h_{\mathrm{sr}} x_{\mathrm{s}}^{\mathrm{i}}+z_{\mathrm{r}}
$$

with $P_{\mathrm{s}}^{\mathrm{i}}$ denoting the power of the information signal. Finally, if the relay correctly decodes the source message, it uses the last time slot to forward the codeword to the destination in $\frac{\theta}{2} T$ channel uses. Thus, the destination's received signal is given by

$$
y_{\mathrm{d}}=\sqrt{P_{\mathrm{r}}^{\mathrm{i}}} h_{\mathrm{rd}} x_{\mathrm{r}}+z_{\mathrm{d}},
$$

where the relay signal $x_{\mathrm{r}}$ follows $\mathcal{C N}(0,1), z_{\mathrm{d}}$ is the additive white Gaussian noise and $P_{\mathrm{r}}^{\mathrm{i}}$ is the relay's information transmission power. Finally, with no loss of generality, we set the variances of the noises equal to 1 and assume the packets to be sufficiently long such that the results are independent of $T$, and it can be removed from the analysis.

We consider an ideal energy consumption model for the source motivated by the fact that the base stations are commonly equipped with considerably stronger power amplifiers than the relays. On the other hand, we adopt linear model for the power consumption of the relay, where the relay's power consumption is modeled by

$$
P_{\text {cons }}=P_{\text {active }}+\nu P_{\mathrm{r}}^{\mathrm{i}}
$$

Here, $\nu \geq 1$ represents the inefficiency of the PA and $P_{\text {active }}$ represents the relay's bias power during data transmission.
This is a well-established model for the PAs [12], [13]. We analyze the system performance for two power allocation modes of the relay:

Non-adaptive transmission power. Here, the relay has a predefined (peak) transmission power. This is an appropriate assumption in the cases with simple relays that can not adopt the transmission power.

Adaptive transmission power. Here, the relay adaptively updates its transmission power to forward the data to the destination with the maximum possible power.

\section{Performance Analysis For Non-ADAPtive} RELAY

Let $E_{\text {proc }}=\frac{\theta}{2} P_{\text {proc }}$ where $P_{\text {proc }}$ is an auxiliary variable to simplify the analytical expressions. With a non-adaptive relay, a constant power $P_{\mathrm{r}}^{\mathrm{i}}$ is used by the relay to forward the information to the destination. Thus, from (4), the total energy consumed by the relay during the second and the third phases is

$$
E_{\mathrm{r}}=\frac{\theta}{2}\left(P_{\text {proc }}+P_{\text {cons }}\right)=\frac{\theta}{2}\left(P_{\text {proc }}+P_{\text {active }}+\nu P_{\mathrm{r}}^{\mathrm{i}}\right) .
$$

In this way, if the the relay's minimum energy is not supplied in Phase 1, the relay does not become active, and circuit outage event occurs. Thus, the probability of circuit outage for nonadaptive relay is

$$
\begin{aligned}
\operatorname{Pr}(\text { Circuit Outage }) & =\operatorname{Pr}\left(g_{\mathrm{sr}} P_{\mathrm{s}}^{\mathrm{e}}(1-\theta)<\frac{\theta}{2}\left(P_{\text {cons }}+P_{\text {proc }}\right)\right) \\
& =1-\exp \left(-\frac{\left(P_{\text {cons }}+P_{\text {proc }}\right) \frac{\theta}{2}}{\lambda_{\text {sr }} P_{\mathrm{s}}^{\mathrm{e}}(1-\theta)}\right) .
\end{aligned}
$$

Here, the last equality holds for Rayleigh fading distribution where the source-relay channel gain $g_{\text {sr }}$ follows exponential distribution with mean $\lambda_{\mathrm{sr}}$. To derive the outage probability, we also need to find the rate outage probability given that the circuit outage event has not occurred. Define the effective SNR as

$$
\gamma_{\mathrm{eff}}=\min \left\{\gamma_{\mathrm{sr}}, \gamma_{\mathrm{rd}}\right\}
$$

where $\gamma_{\mathrm{sr}}=g_{\mathrm{sr}} P_{\mathrm{s}}^{\mathrm{i}}$ and $\gamma_{\mathrm{rd}}=g_{\mathrm{rd}} P_{\mathrm{r}}^{\mathrm{i}}$ are the relay's and the destination's SNRs, respectively. In this way, representing the code rate by $R$, the rate outage probability is found as

$$
\begin{aligned}
& \operatorname{Pr}(\text { Rate Outage } \mid \text { No Circuit Outage }) \\
& =1-\operatorname{Pr}\left(\frac{\theta}{2} \log \left(1+\gamma_{\mathrm{eff}}\right) \geq R \mid \text { No Circuit Outage }\right) \\
& =1-\operatorname{Pr}\left(g_{\mathrm{sr}} P_{\mathrm{s}}^{\mathrm{i}} \geq \gamma, g_{\mathrm{rd}} P_{\mathrm{r}}^{\mathrm{i}} \geq \gamma \mid g_{\mathrm{sr}} \geq \frac{\frac{\theta}{2}\left(P_{\mathrm{cons}}+P_{\text {proc }}\right)}{P_{\mathrm{s}}^{\mathrm{e}}(1-\theta)}\right) \\
& = \begin{cases}1-\exp \left(-\frac{\gamma}{\lambda_{\mathrm{rd}} P_{\mathrm{r}}^{\mathrm{i}}}\right) & \frac{P_{\text {proc }}+P_{\text {cons }}}{P_{\mathrm{s}}^{\mathrm{e}}(1-\theta)} \geq \frac{\gamma}{P_{\mathrm{s}}^{\mathrm{i}} \frac{\theta}{2}} \\
1-\frac{\exp \left(-\frac{\gamma}{\lambda_{\mathrm{sr}} P_{\mathrm{s}}^{\mathrm{i}}}\right) \exp \left(-\frac{\gamma}{\lambda_{\mathrm{rd}} P_{\mathrm{r}}^{\mathrm{i}}}\right)}{\exp \left(-\frac{\theta}{2}\left(P_{\mathrm{cons}}+P_{\text {proc }}\right)\right.} & \frac{P_{\text {proc }}+P_{\text {cons }}}{P_{\mathrm{sr}} P_{\mathrm{s}}^{\mathrm{e}}(1-\theta)} \leq \frac{\gamma}{P_{\mathrm{s}}^{\mathrm{i}}(1-\theta)}\end{cases}
\end{aligned}
$$

where the last equality holds for Rayleigh fading conditions of the relay-destination link with mean $\mathbb{E}\left[g_{\mathrm{rd}}\right]=\lambda_{\text {rd }}$ and 
$\gamma=\exp \left(\frac{2 R}{\theta}\right)-1$ is an auxiliary variable to simplify the expressions. Using (6), (8) and some manipulations, the outage probability for the non-adaptive power allocation is found as

$$
\begin{aligned}
& \operatorname{Pr}(\text { outage }) \\
& = \begin{cases}1-e^{-\left(\frac{\left(P_{\text {cons }}+P_{\text {proc }}\right) \frac{\theta}{2}}{\lambda_{\mathrm{sr}} P_{\mathrm{s}}^{\mathrm{e}}(1-\theta)}+\frac{\gamma}{\lambda_{\mathrm{rd}} P_{\mathrm{r}}^{\mathrm{i}}}\right)} & \frac{P_{\mathrm{proc}}+P_{\text {cons }}}{P_{\mathrm{s}}^{\mathrm{e}}(1-\theta)} \geq \frac{\gamma}{P_{\mathrm{s}}^{\mathrm{i}} \frac{\theta}{2}} \\
1-e^{-\left(\frac{\gamma}{\lambda_{\mathrm{sr}} P_{\mathrm{s}}^{\mathrm{i}}}+\frac{\gamma}{\lambda_{\mathrm{rd}} P_{\mathrm{r}}^{\mathrm{i}}}\right)} & \frac{P_{\mathrm{proc}}+P_{\text {cons }}}{P_{\mathrm{s}}^{\mathrm{e}}(1-\theta)} \leq \frac{\gamma}{P_{\mathrm{s}}^{\mathrm{i}} \frac{\theta}{2}}\end{cases}
\end{aligned}
$$

Finally, the source expected consumed energy is obtained by

$$
\begin{aligned}
\bar{E}_{\mathrm{s}} & =P_{\mathrm{s}}^{\mathrm{e}}(1-\theta)+P_{\mathrm{s}}^{\mathrm{i}} \frac{\theta}{2} \operatorname{Pr}(\text { source becomes active in Phase } 2) \\
& =P_{\mathrm{s}}^{\mathrm{e}}(1-\theta)+P_{\mathrm{s}}^{\mathrm{i}} \frac{\theta}{2} \exp \left(-\frac{\left(P_{\text {cons }}+P_{\text {proc }}\right) \frac{\theta}{2}}{\lambda_{\mathrm{sr}} P_{\mathrm{s}}^{\mathrm{e}}(1-\theta)}\right)
\end{aligned}
$$

Thus, considering a total energy constraint $\bar{E}_{\mathrm{s}} \leq E_{\max }$, the energy-constrained outage minimization problem can be written as

$$
\begin{array}{ll}
\underset{\theta, P_{\mathrm{s}}^{\mathrm{i}}, P_{\mathrm{s}}^{\mathrm{e}}}{\operatorname{minimize}} & \operatorname{Pr}(\text { outage }) \\
\text { subject to } & \bar{E}_{\mathrm{s}} \leq E_{\max }=P_{\max } T,
\end{array}
$$

with $P_{\mathrm{s}}^{\mathrm{e}}, P_{\mathrm{s}}^{\mathrm{i}}$ and $\theta$ being the optimization parameters. Since we set $T=1$, the $E_{\max }$ and $P_{\max }$ can be used interchangeably.

\section{A. Optimal Fixed Time Sharing}

In this section, we minimize the outage probability in (9) with respect to $\theta$ in the cases with a peak power constraint at the source, i.e., $P_{\mathrm{s}}^{\mathrm{e}}=P_{\mathrm{s}}^{\mathrm{i}}=P_{0}$, which is solved in Theorem 1 .

Theorem 1. Optimal time sharing between the power and information signals is given by

$$
\theta^{*}= \begin{cases}\theta_{1}^{*} & \frac{2\left(1-\theta_{1}^{*}\right)\left(\exp \left(\frac{2 R}{\theta_{1}^{*}}\right)-1\right)}{\theta_{1}^{*}} \leq P_{\mathrm{cons}}+P_{\mathrm{proc}} \\ \theta_{2}^{\star} & \text { O.W }\end{cases}
$$

where $\theta_{1}^{*}=\frac{R}{R+\mathcal{W}\left(\frac{\sqrt{\lambda_{\mathrm{rd}} P_{\mathrm{r}}^{\mathrm{i}}\left(P_{\mathrm{cons}}+P_{\mathrm{proc}}\right) R}}{2 \sqrt{\lambda_{\mathrm{sr}} P_{0}} \exp (R)}\right)}, \quad \theta_{2}^{\star} \simeq$ $\frac{2 R}{2 R+\mathcal{W}\left(R\left(P_{\text {cons }}+P_{\text {proc }}\right) \exp (-2 R)\right)} \quad$ and $\mathcal{W}(x)$ denotes the Lambert W function [14].

Proof. Let $f_{1}(\theta)=\exp \left(-\frac{\gamma}{\lambda_{\mathrm{rd}} P_{\mathrm{r}}^{\mathrm{i}}}\right) \exp \left(-\frac{\left(P_{\text {cons }}+P_{\text {proc }}\right) \frac{\theta}{2}}{\lambda_{\mathrm{sr}} P_{0}(1-\theta)}\right)$ and $f_{2}(\theta)=\exp \left(-\frac{\gamma}{\lambda_{\mathrm{sr}} P_{0}}\right) \exp \left(-\frac{\gamma}{\lambda_{\mathrm{rd}} P_{\mathrm{r}}^{\mathrm{i}}}\right)$. Also, we define $g_{1}(\theta)=\log \left(f_{1}(\theta)\right)$ and $g_{2}(\theta)=\log \left(f_{2}(\theta)\right)$. Taking the second derivative of $g_{1}(\theta), g_{2}(\theta)$ functions, it is straightforward to show that they are concave functions in $\theta$. Therefore, the optimal time scheduling is found by setting the derivative of the objective functions equal to zero. Particularly, using $f_{1}(\theta)$, we have

$$
\begin{gathered}
\theta_{1}^{*}=\underset{0 \leq \theta \leq 1}{\arg \max }\left\{g_{1}(\theta)=-\frac{\gamma}{\lambda_{\mathrm{rd}} P_{\mathrm{r}}^{\mathrm{i}}}-\frac{\left(P_{\text {cons }}+P_{\mathrm{proc}}\right) \frac{\theta}{2}}{\lambda_{\mathrm{sr}} P_{0}(1-\theta)}\right\} \\
\stackrel{(\mathrm{a})}{=} \underset{0 \leq \theta \leq 1}{\arg }\left\{\frac{P_{\text {cons }}+P_{\mathrm{proc}}}{2 \lambda_{\mathrm{sr}} P_{0}(1-\theta)^{2}}=\frac{2 R}{\lambda_{\mathrm{rd}} P_{\mathrm{r}}^{\mathrm{i}}} \frac{\exp \left(\frac{2 R}{\theta}\right)}{\theta^{2}}\right\} \\
\Rightarrow \theta_{1}^{*}=\frac{R}{R+\mathcal{W}\left(\frac{\sqrt{\lambda_{\mathrm{rd}} P_{\mathrm{r}}^{\mathrm{i}}\left(P_{\text {cons }}+P_{\text {proc }}\right) R}}{2 \sqrt{\lambda_{\mathrm{sr}} P_{0}} \exp (R)}\right)} .
\end{gathered}
$$

where (a) is obtained by setting $\frac{\mathrm{d} g_{1}}{\mathrm{~d} \theta}=0$ and the last equality is obtained by some manipulations and the definition of Lambert $\mathcal{W}$ function. For $f_{2}(\theta)$, we have

$$
\theta_{2}^{*}=\underset{0 \leq \theta \leq 1}{\arg \max }\left\{g_{2}(\theta)=-\frac{\gamma}{\lambda_{\mathrm{sr}} P_{0}}-\frac{\gamma}{\lambda_{\mathrm{rd}} P_{\mathrm{r}}^{\mathrm{i}}}\right\}
$$

Since $g_{2}(\theta)$ is an increasing function of $\theta$, its minimum value is given by the boundary of the branches in (9), i.e.,

$$
\begin{aligned}
\theta_{2}^{*} & =\underset{0 \leq \theta \leq 1}{\arg }\left\{\frac{\gamma}{\frac{\theta}{2}}=\frac{P_{\text {cons }}+P_{\text {proc }}}{(1-\theta)}\right\} \\
& =\underset{0 \leq \theta \leq 1}{\arg }\left\{\exp \left(\frac{2 R}{\theta}\right)-1-\frac{P_{\text {cons }}+P_{\text {proc }}}{2} \frac{\theta}{1-\theta}=0\right\} .
\end{aligned}
$$

Defining $y(\theta)=\exp \left(\frac{2 R}{\theta}\right)-1-\frac{P_{\text {cons }}+P_{\text {proc }}}{2} \frac{\theta}{1-\theta}$, it is straightforward to show that $\lim _{\theta \rightarrow 0} y(\theta)=+\infty, \lim _{\theta \rightarrow 1} y(\theta)=-\infty$ and $y(\theta)$ is a decreasing function of $\theta \in[0,1]$. Therefore, the solution of $y(\theta)=0$ will be unique for any values of $P_{\text {cons }}, P_{\text {proc }}, R$. Unfortunately, to the best of authors knowledge, there is no closed-form solution for $y(\theta)=0$. Thus, considering the moderate/large values of $R, \theta_{2}^{*}$ is approximately given by

$$
\begin{aligned}
& \theta_{2}^{*} \simeq \arg _{\theta}\left\{\exp \left(\frac{2 R}{\theta}\right)-\frac{P_{\text {cons }}+P_{\text {proc }}}{2} \frac{\theta}{1-\theta}=0\right\} \\
& \Rightarrow \theta_{2}^{*} \simeq \frac{2 R}{2 R+\mathcal{W}\left(R\left(P_{\text {cons }}+P_{\text {proc }}\right) \exp (-2 R)\right)},
\end{aligned}
$$

Note that branching condition in (9) can be rephrased in term of $\theta$ as $\theta \in\left[\theta_{2}^{\star}, 1\right]$ and $\theta \in\left[0, \theta_{2}^{\star}\right]$ for upper and lower branch, respectively. Let the $\frac{\left(1-\theta_{1}^{*}\right)\left(\exp \left(\frac{2 R}{\theta_{1}^{*}}\right)-1\right)}{\frac{\theta_{1}^{*}}{2}} \geq P_{\text {cons }}+P_{\text {proc }}$. By calculating the first derivative $\frac{f_{1}^{2}}{(\theta)}$ and $f_{2}(\theta)$, it can be proved that $f_{1}(\theta)$ is strictly decreasing function in $\left[\theta_{2}^{\star}, 1\right]$ and $f_{2}(\theta)$ is strictly increasing in $\left[0, \theta_{2}^{\star}\right]$. Thus, the optimal $\theta$ for this case is $\theta_{2}^{\star}$ (lower branch of (12)). In the other case where $\frac{\left(1-\theta_{1}^{*}\right)\left(\exp \left(\frac{2 R}{\theta_{1}^{*}}\right)-1\right)}{\frac{\theta_{1}^{*}}{2}} \leq P_{\text {cons }}+P_{\text {proc }}$, we can use (13) to show that $\theta_{1}^{*} \in\left[\theta_{2}^{*}, 1\right]$. In this case, $f_{1}(\theta)$ is increasing in $\left[\theta_{2}^{\star}, \theta_{1}^{\star}\right]$ and decreasing in $\left[\theta_{1}^{\star}, 1\right]$ also $f_{2}(\theta)$ is strictly increasing in $\left[0, \theta_{2}^{\star}\right]$. Thus, the optimal value for $\theta$ is $\theta_{1}^{\star}$ ( the upper branch of (12)).

Although the optimal point in the lower branch of (12) is derived for moderate/large codeword rate, Fig. 2 indicates that the approximation is very tight for broad ranges of codeword 
rate. Moreover, the optimal time sharing is independent of $P_{0}$ in the lower branch of (12). Finally, at high SNRs, since $\lim _{x \rightarrow 0} \mathcal{W}(x)=0$, the optimal time sharing in the upper branch of (12) converges into 1 , as expected.

\section{B. Optimal Power Allocation}

Considering given fractional signal duration, i.e., $\theta$, the optimal power allocation at the source between energy and information signals is provided in Theorem 2 .

Theorem 2. The outage-optimized source power allocation for the energy and information transfer signals is given by

$$
\begin{aligned}
& \left(P_{\mathrm{s}}^{\mathrm{e}}\right)^{\star}=\frac{P_{\max } \theta}{2(1-\theta)}\left(P_{\text {proc }}+P_{\text {cons }}\right)\left[\left(P_{\text {proc }}+P_{\text {cons }}\right) \theta\right. \\
& \left.+\lambda_{\mathrm{sr}} P_{\max } \mathcal{W}\left(\frac{\gamma \theta}{\lambda_{\mathrm{sr}} P_{\max }} \exp \left(-\frac{\left(P_{\text {proc }}+P_{\text {cons }}\right) \frac{\theta}{2}}{\lambda_{\mathrm{sr}} P_{\text {max }}}\right)\right)\right]^{-1}, \\
& \left(P_{\mathrm{s}}^{\mathrm{i}}\right)^{\star}=\frac{P_{\max }-\left(P_{\mathrm{s}}^{\mathrm{e}}\right)^{\star}(1-\theta)}{\frac{\theta}{2} \exp \left(-\frac{1}{\lambda_{\mathrm{sr}}} \frac{P_{\text {proc }}+P_{\mathrm{cons}}}{\left(P_{\mathrm{s}}^{\mathrm{e}}\right)^{\star}} \frac{\frac{\theta}{2}}{1-\theta}\right)},
\end{aligned}
$$

where $\mathcal{W}(x)$ denotes the Lambert $\mathrm{W}$ function [14].

Proof. It is straightforward to prove that the energy constraint of (11) should hold with equality in the optimal case. Considering the equality, the branching condition of (9) is rephrased in term of $P_{\mathrm{s}}^{\mathrm{e}}$ as

$$
\begin{aligned}
& \frac{\gamma}{\frac{\theta}{2} \exp \left(-\frac{1}{\lambda_{\mathrm{sr}}} \frac{P_{\mathrm{proc}}+P_{\text {cons }}}{P_{\mathrm{s}}^{\mathrm{e}}} \frac{\frac{\theta}{2}}{1-\theta}\right)} \frac{\theta}{2} \leq \frac{P_{\text {cons }}+P_{\text {proc }}}{P_{\mathrm{s}}^{\mathrm{e}}(1-\theta)} \\
& \Rightarrow P_{\mathrm{s}}^{\mathrm{e}} \leq P_{\max } \frac{\theta}{2(1-\theta)}\left(P_{\text {proc }}+P_{\text {cons }}\right)\left[\left(P_{\text {proc }}+P_{\text {cons }}\right) \theta\right. \\
& \left.+\lambda_{\mathrm{sr}} P_{\max } \mathcal{W}\left(\frac{\gamma \theta}{\lambda_{\mathrm{sr}} P_{\max }} \exp \left(-\frac{\left(P_{\text {proc }}+P_{\text {cons }}\right) \frac{\theta}{2}}{\lambda_{\mathrm{sr}} P_{\max }}\right)\right)\right]^{-1} \\
& \doteq \hat{P}_{\mathrm{s}}^{\mathrm{e}} .
\end{aligned}
$$

Also, according to the energy constraint of (11) and $P_{\mathrm{s}}^{\mathrm{e}} \geq 0$, we have $P_{\mathrm{s}}^{\mathrm{e}} \leq \frac{P_{\max }}{1-\theta}$, because $\exp \left(-\frac{\gamma}{\lambda_{\mathrm{rd}} P_{\mathrm{r}}^{\mathrm{i}}}\right) \exp \left(-\frac{\left(P_{\text {cons }}+P_{\text {proc }}\right) \frac{\theta}{2}}{\lambda_{\mathrm{sr}} P_{\mathrm{s}}^{\mathrm{e}}(1-\theta)}\right)$ is an increasing function of $P_{s}^{e}$, and the optimal value of $P_{s}^{e}$ in the first branch of (9) is given by

$$
\left(P_{\mathrm{s}}^{\mathrm{e}}\right)^{\star}=\min \left\{\frac{P_{\max }}{1-\theta}, \hat{P}_{\mathrm{s}}^{\mathrm{e}}\right\} .
$$

Then, as $\mathcal{W}(x)>0$ for $x>0$, we have

$$
\hat{P_{\mathrm{s}}^{\mathrm{e}}} \leq \frac{P_{\mathrm{max}}}{1-\theta},
$$

and, from (18), (19) and (20), the optimal values of $P_{\mathrm{s}}^{\mathrm{e}}$ and $P_{\mathrm{s}}^{\mathrm{i}}$ for the first branch of (9) are given by (17). For the second branch of (9), on the other hand, since the objective function $\exp \left(-\frac{\gamma}{\lambda_{\mathrm{sr}} P_{\mathrm{s}}^{\mathrm{1}}}\right) \exp \left(-\frac{\gamma}{\lambda_{\mathrm{rd}} P_{\mathrm{r}}^{\mathrm{i}}}\right)$ is a decreasing function of $P_{\mathrm{s}}^{\mathrm{e}}$, the optimal value of $P_{\mathrm{s}}^{\mathrm{e}}$ is given by the boundary of the feasible set which leads to the same value as (17). That is, the optimal point for $\left[\left(P_{\mathrm{s}}^{\mathrm{e}}\right)^{\star} ;\left(P_{\mathrm{s}}^{\mathrm{i}}\right)^{\star}\right]$ is the same for both objective functions. Thus, the outage-optimized power allocation rule is given by (17) as stated in the theorem.

Using (17) and $\lim _{x \rightarrow 0^{+}} \mathcal{W}^{\prime}(x)=1$ [14], for $P_{\max } \rightarrow \infty$, we have

$$
\left(P_{\mathrm{s}}^{\mathrm{e}}\right)^{\star} \simeq\left(\frac{\left(P_{\text {proc }}+P_{\text {cons }}\right)}{2(1-\theta)\left(\exp \left(\frac{2 R}{\theta}\right)+P_{\text {proc }}+P_{\text {cons }}\right)}\right) P_{\text {max }},
$$

which implies that, the optimal power for energy transfer increases linearly with $P_{\max }$ and also decreases exponentially with codeword rate.

\section{ON THE EFFECT OF RELAY'S POWER ADAPTATION}

In this section, we assume that the relay can adaptively update the transmission power in the third phase and it uses all available energy to forward the source message with maximum power. Thus, the relay transmission power is obtained by

$$
\begin{aligned}
& \left(P_{\text {active }}+\nu P_{\mathrm{r}}^{\mathrm{i}}\right) \frac{\theta}{2}=g_{\mathrm{sr}} P_{\mathrm{s}}^{\mathrm{e}}(1-\theta)-P_{\text {proc }} \frac{\theta}{2} \\
& \Rightarrow P_{\mathrm{r}}^{\mathrm{i}}=\frac{g_{\mathrm{sr}} P_{\mathrm{s}}^{\mathrm{e}}(1-\theta)-\left(P_{\text {proc }}+P_{\text {active }}\right) \frac{\theta}{2}}{\nu \frac{\theta}{2}} .
\end{aligned}
$$

Thus, using adaptive power allocation for the relay, the outage probability is rephrased as (23) shown on the top of the next page. In (23), $Q=\max \left\{0, \frac{\lambda_{\mathrm{rd}}}{\nu} \frac{P_{\mathrm{s}}^{\mathrm{e}}(1-\theta)}{P_{\mathrm{s}}^{\mathrm{i}} \frac{\theta}{2}}-\frac{\left(P_{\text {proc }}+P_{\text {active }}\right) \lambda_{\mathrm{rd}}}{\nu \gamma}\right\}$ and the last equality comes from the variable transformation $u=\frac{\lambda_{\mathrm{rd}}\left(x P_{\mathrm{s}}^{\mathrm{e}}(1-\theta)-\left(P_{\text {proc }}+P_{\text {active }}\right) \frac{\theta}{2}\right)}{\frac{\theta}{2} \nu \gamma_{0}}$. In this way, the integral in (23) needs to be calculated in the following two cases: $\frac{\gamma}{P_{\mathrm{s}}^{\mathrm{i}}} \geq \frac{\left(P_{\text {proc }}+P_{\text {active }}\right) \frac{\theta}{2}}{P_{\mathrm{s}}^{\mathrm{e}}(1-\theta)}$ and $\frac{\gamma}{P_{\mathrm{s}}^{\mathrm{i}}} \leq \frac{\left(P_{\text {proc }}+P_{\text {active }}\right) \frac{\theta}{2}}{P_{\mathrm{s}}^{\mathrm{e}}(1-\theta)}$. Considering the case with $\gamma \leq\left(P_{\text {proc }}+P_{\text {active }}\right) \frac{\frac{\theta}{2} P_{\mathrm{s}}^{\mathrm{i}}}{(1-\theta) P_{\mathrm{s}}^{\mathrm{e}}}$, we have

$$
\begin{aligned}
& \operatorname{Pr}(\text { outage })=1-\frac{\frac{\theta}{2} \nu \frac{\gamma}{\lambda_{\mathrm{rd}}}}{\lambda_{\mathrm{sr}} P_{\mathrm{s}}^{\mathrm{e}}(1-\theta)} \exp \left(-\frac{\left(P_{\text {proc }}+P_{\text {active }}\right) \frac{\theta}{2}}{\lambda_{\mathrm{sr}} P_{\mathrm{s}}^{\mathrm{e}}(1-\theta)}\right) \\
& \times \int_{0}^{\infty} \exp \left(-\frac{1}{u}\right) \exp \left(-\frac{1}{\lambda_{\mathrm{sr}}} \frac{u \frac{\theta}{2} \nu \frac{\gamma}{\lambda_{\mathrm{rd}}}}{P_{\mathrm{s}}^{\mathrm{e}}(1-\theta)}\right) \mathrm{d} u=\sqrt{\frac{2 \theta \nu \frac{\gamma}{\lambda_{\mathrm{rd}}}}{\lambda_{\mathrm{sr}} P_{\mathrm{s}}^{\mathrm{e}}(1-\theta)}} \\
& \times \exp \left(-\frac{\left(P_{\mathrm{proc}}+P_{\text {active }}\right) \frac{\theta}{2}}{\lambda_{s r} P_{\mathrm{s}}^{\mathrm{e}}(1-\theta)}\right) K_{1}\left(\sqrt{\frac{2 \theta \nu \frac{\gamma}{\lambda_{\mathrm{rd}}}}{\lambda_{\mathrm{sr}} P_{\mathrm{s}}^{\mathrm{e}}(1-\theta)}}\right),
\end{aligned}
$$

where the last equality is obtained by the definition of the modified Bessel function of second kind in [15, Eq. 3.324.1]. On the other hand, if $\gamma \geq\left(P_{\text {proc }}+P_{\text {active }}\right) \frac{\frac{\theta}{2} P_{\mathrm{s}}^{\mathrm{i}}}{(1-\theta) P_{\mathrm{s}}^{\mathrm{e}}}$, the outage probability is given by

$$
\begin{aligned}
& \operatorname{Pr}(\text { outage })=1-\frac{\frac{\theta}{2} \nu \frac{\gamma}{\lambda_{\mathrm{rd}}}}{\lambda_{\mathrm{sr}} P_{\mathrm{s}}^{\mathrm{e}}(1-\theta)} \exp \left(-\frac{\left(P_{\text {proc }}+P_{\text {active }}\right) \frac{\theta}{2}}{\lambda_{\mathrm{sr}} P_{\mathrm{s}}^{\mathrm{e}}(1-\theta)}\right) \\
& \times \int_{L_{b}}^{\infty} \exp \left(-\frac{1}{u}\right) \exp \left(-\frac{1}{\lambda_{\mathrm{sr}}} \frac{u \frac{\theta}{2} \nu \frac{\gamma}{\lambda_{\mathrm{rd}}}}{P_{\mathrm{s}}^{\mathrm{e}}(1-\theta)}\right) \mathrm{d} u \simeq 1-\frac{c_{1}}{P_{\mathrm{s}}^{\mathrm{e}}} \\
& \times \exp \left(-\frac{c_{2}}{P_{\mathrm{s}}^{\mathrm{e}}}\right) \int_{L_{b}}^{\infty} \sum_{n=0}^{N} \frac{(-1)^{n}}{n !} \frac{1}{u^{n}} \exp \left(-\frac{c_{1}}{\left.P_{\mathrm{s}}^{\mathrm{e}} u\right) \mathrm{d} u}\right.
\end{aligned}
$$




$$
\begin{aligned}
\operatorname{Pr}(\text { Outage }) & =1-\int_{0}^{\infty} \int_{0}^{\infty} \operatorname{Pr}\left(x \geq \frac{\left(P_{\mathrm{proc}}+P_{\text {active }}\right) \frac{\theta}{2}}{P_{\mathrm{s}}^{\mathrm{e}}(1-\theta)}, x P_{\mathrm{s}}^{\mathrm{i}} \geq \gamma, y P_{\mathrm{r}}^{\mathrm{t}} \geq \gamma \mid g_{\mathrm{sr}}=x, g_{\mathrm{rd}}=y\right) f_{g_{\mathrm{sr}}}(x) f_{g_{\mathrm{rd}}}(y) \mathrm{d} x \mathrm{~d} y \\
& =1-\frac{\frac{\theta}{2} \nu \frac{\gamma}{\lambda_{\mathrm{rd}}}}{\lambda_{\mathrm{sr}} P_{\mathrm{s}}^{\mathrm{s}}(1-\theta)} \int_{Q}^{\infty} \exp \left(-\frac{1}{u}\right) \exp \left(-\frac{1}{\lambda_{\mathrm{sr}}} \frac{u \frac{\theta}{2} \nu \frac{\gamma}{\lambda_{\mathrm{rd}}}+\left(P_{\text {proc }}+P_{\text {active }}\right) \frac{\theta}{2}}{P_{\mathrm{s}}^{\mathrm{e}}(1-\theta)}\right) \mathrm{d} u .
\end{aligned}
$$

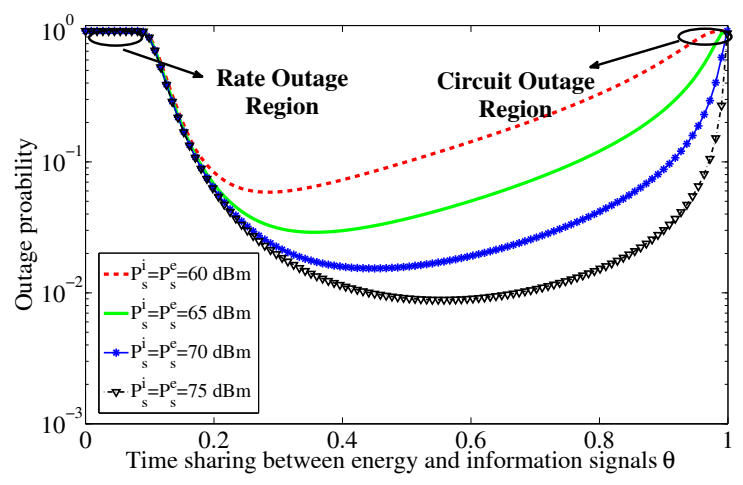

Fig. 1: Outage probability versus time sharing between energy and information signal for different source transmission, $\lambda_{\mathrm{sr}}=$ $\lambda_{\mathrm{rd}}=1, \nu=2, P_{\text {proc }}=23.01(\mathrm{dBm}), P_{\text {active }}=29.03(\mathrm{dBm})$, $P_{\mathrm{r}}^{\mathrm{i}}=55(\mathrm{dBm})$.

$$
=1-\frac{c_{1}}{L_{b} P_{\mathrm{s}}^{\mathrm{e}}} \exp \left(-\frac{c_{2}}{P_{\mathrm{s}}^{\mathrm{e}}}\right) \sum_{n=0}^{N}\left(-L_{b}\right)^{-n} \mathrm{E}_{n}\left(-\frac{c_{1} L_{b}}{P_{\mathrm{s}}^{\mathrm{e}}}\right),
$$

for $\forall N \geq 1$, where $L_{b}=\frac{\lambda_{\mathrm{rd}}}{\nu} \frac{P_{\mathrm{s}}^{\mathrm{e}}(1-\theta)}{P_{\mathrm{s}}^{\mathrm{i}} \frac{\theta}{2}}-\frac{\left(P_{\text {proc }}+P_{\text {active }}\right) \lambda_{\mathrm{rd}}}{\nu \gamma}, c_{1}=$ $\frac{\frac{\theta}{2} \nu \frac{\gamma}{\lambda_{\mathrm{rd}}}}{\lambda_{\mathrm{sr}}(1-\theta)}, c_{2}=\frac{\left(P_{\text {proc }}+P_{\text {active }}\right) \frac{\theta}{2}}{\lambda_{\mathrm{rr}}(1-\theta)}$, and the last equality is obtained by the definition of generalized exponential integral in [15, Eq. 8.211.1]. From (24) and (25), the overall outage probability for the power-adaptive relay is given by

$$
\operatorname{Pr}(\text { outage }) \simeq\left\{\begin{array}{ll}
Y_{1} & \frac{\gamma}{P_{\mathrm{s}}^{\mathrm{i}}} \leq \frac{\left(P_{\mathrm{proc}}+P_{\text {active }}\right) \frac{\theta}{2}}{P_{\mathrm{s}}^{\mathrm{e}}(1-\theta)} \\
Y_{2} & \frac{\gamma}{P_{\mathrm{s}}^{\mathrm{i}}} \geq \frac{\left(P_{\text {proc }}+P_{\text {active }}\right) \frac{\theta}{2}}{P_{\mathrm{s}}^{\mathrm{e}}(1-\theta)}
\end{array},\right.
$$

where $Y_{1}=1-2 \sqrt{\frac{c_{1}}{P_{\mathrm{s}}^{\mathrm{e}}}} \exp \left(-\frac{c_{2}}{P_{\mathrm{s}}^{\mathrm{e}}}\right) K_{1}\left(2 \sqrt{\frac{c_{1}}{P_{\mathrm{s}}^{\mathrm{e}}}}\right)$ and $Y_{2}=$ $1-\frac{c_{1}}{L_{b} P_{\mathrm{s}}^{\mathrm{e}}} \exp \left(-\frac{c_{2}}{P_{\mathrm{s}}^{\mathrm{e}}}\right) \sum_{n=0}^{N}\left(-L_{b}\right)^{-n} E_{n}\left(-\frac{c_{1} L_{b}}{P_{\mathrm{s}}^{\mathrm{e}}}\right)$.

In Section V, we validate the accuracy of our analytical results by comparing them with the corresponding exact values that are obtained by simulations.

\section{Simulation Results}

In all figures, we consider fading channels with $\lambda_{\mathrm{sr}}=\lambda_{\mathrm{rd}}=$ 1 , and we do not consider the large scale fading. Also, we set $P_{\text {proc }}=200 \mathrm{~mW}=23.01 \mathrm{dBm}$, and $P_{\text {active }}=800 \mathrm{~mW}=29.03$ $\mathrm{dBm}$ [12]. The slope in the power consumption model in (4) is set to $\nu=2$, which is typical for the class-AB power amplifier.

Figure 1 shows the outage probabilities of the non-adaptive power allocation scheme, given in (9), versus the time sharing between the lengths of the power and information signals, i.e., $\theta$, for different source transmission powers. We have

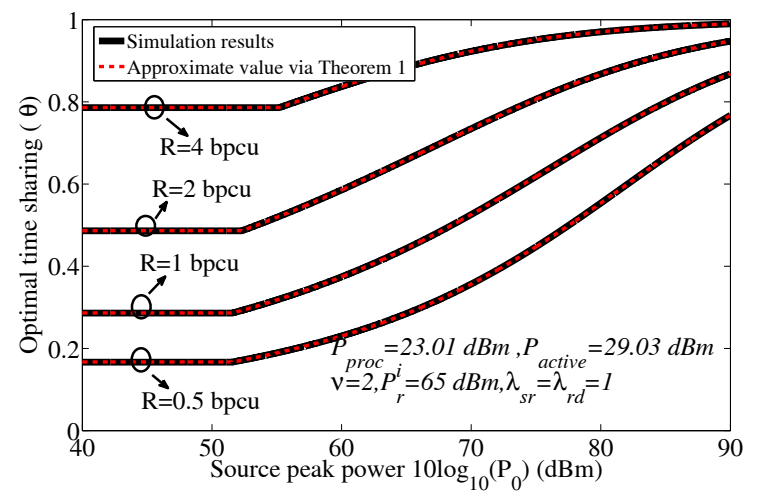

Fig. 2: Optimal time sharing versus source transmission power.

set $P_{\mathrm{r}}^{\mathrm{i}}=55 \mathrm{dBm}$. Based on the segmentation of each communication block, the outage event for small $\theta$ is due to the rate outage event, since for $\theta \rightarrow 0$, a small amount of the block time is allocated for data transmission. On the other hand, for large values of $\theta$, the circuit outage event occurs since energy harvesting period in each block is $1-\theta$. Hereby, we can define "circuit outage region" and "rate outage region" as indicated in Fig. 1. In the rate outage region, the outage probability is dependent on the codeword rate. On the other hand, in the circuit outage region, the outage probability is dependent on the source transmission power. As a result, the rate outage regions are similar for the curves in Fig. 1.

Figure 2 considers the optimal time sharing for a predetermined source peak power, which is given in Theorem 1, and compares the results with the ones derived by exhaustive search. As the codeword rate increases, the optimal time sharing increases since the transmission rate is proportional to $\theta$. Optimal time sharing has two regions. In the first region, it is independent of the source transmission power, which is given in the lower branch of (12). In the second region, it is an increasing function of the source transmission power and approaches one as the source transmission power increases. The approximation technique of Theorem 1 is very tight for a broad range of source transmission power.

Figs. 3 and 4 compare the performance of the different schemes considered in this paper, in terms of outage probability and throughput, respectively. In Fig. 3, we have set the relay transmission power for non-adaptive schemes to $P_{\mathrm{r}}^{\mathrm{i}}=50$ $\mathrm{dBm}$, the time sharing for the adaptive power allocation for the relay is set to $\theta=0.4$, and for non-adaptive with fixed time sharing is set to its optimal value given in Theorem 1 . From Fig. 3, the high-SNR outage probability of the fixed 


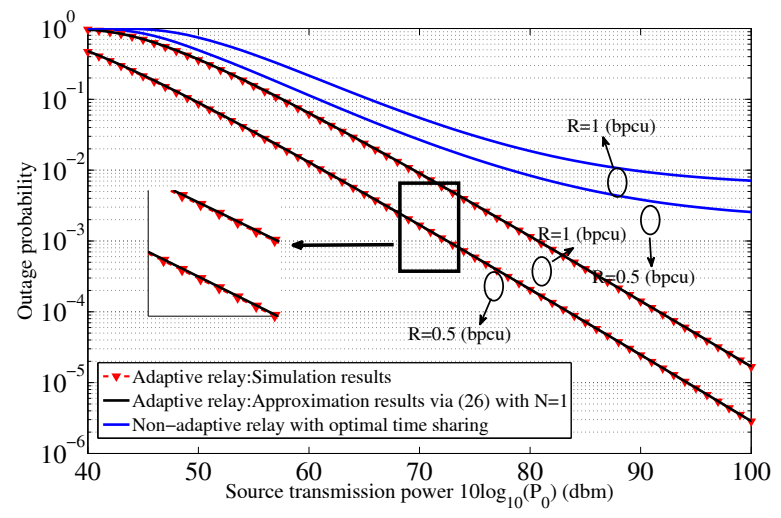

Fig. 3: Comparison of the different protocols in term of outage probability, $\lambda_{\mathrm{sr}}=\lambda_{\mathrm{rd}}=1, P_{\text {active }}=29.03(\mathrm{dBm}), \nu=2$, for the non-adaptive scheme $P_{\mathrm{r}}^{\mathrm{i}}=50(\mathrm{dBm})$, for the adaptive scheme $\theta=0.4$.

time sharing scheme converges to a non-zero limit, because at high-SNR, the outage probability of the non-adaptive system is mostly due to the rate outage in Phase 3, and it is independent of the source transmission power. Additionally, the relay with the adaptive power allocation outperforms the other scenarios. Also, as seen in this figure, the approximation approach of (26) is very tight for a broad range of SNRs/parameter settings, such that 2 terms in (26) provide accurate results.

Moreover, Fig. 4 compares the throughput for different protocols and parameter settings. The throughput of a given protocol with codeword rate $R$ and outage probability $\operatorname{Pr}$ (outage) is defined by $\mathcal{T}=R(1-\operatorname{Pr}$ (outage)). As demonstrated in this figure, the adaptive power allocation in the relay has better performance compared to other protocols. Also, setting time sharing or power allocation at the source to its optimal value significantly increases the system throughput. Furthermore, with the parameter settings of the figure, the relay network with optimal time sharing but uniform power allocation at the source has higher throughput than the system with fixed time sharing but optimal power allocation at the source.

\section{CONCLUSION}

Considering imperfect power consumption models for the relay, we studied the outage probability and the throughput of the relay networks with joint information and energy transfer. We analyzed the system performance for two power allocation schemes of the relay, namely non-adaptive and adaptive transmission powers. For the non-adaptive scheme, we derived the optimal time sharing and optimal power allocation with an expected total energy consumption constraint. Then, we analyzed the outage probability in the cases with the adaptive power allocation by the relay. As demonstrated both analytically and numerically, the optimal power for the energy transfer signal increases linearly with the total energy of the source and decreases exponentially with the codeword rate. Finally, our results demonstrate the joint energy and information transfer as an effective technique towards green communication.

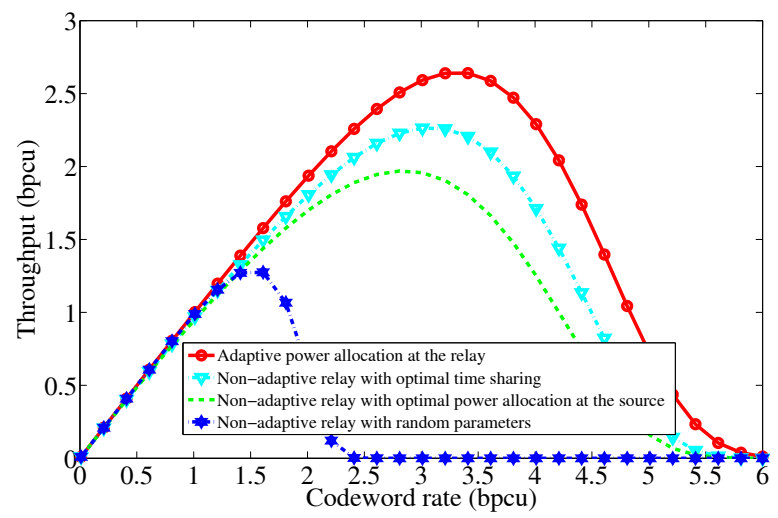

Fig. 4: Comparison of different protocols in term of throughput, $\lambda_{\mathrm{sr}}=\lambda_{\mathrm{rd}}=1, P_{\text {active }}=29.03(\mathrm{dBm}), \nu=2$, for the non-adaptive scheme $P_{\mathrm{r}}^{\mathrm{i}}=40(\mathrm{dBm})$, for the adaptive scheme $\theta=0.4$ and source maximum energy to $60(\mathrm{dBm})$.

\section{REFERENCES}

[1] X. Lu, P. Wang, D. Niyato, D. I. Kim, and Z. Han, "Wireless networks with RF energy harvesting: A contemporary survey," IEEE Commun. Surveys Tuts., vol. 17, no. 2, pp. 757 - 789, May 2015.

[2] L. Varshney, "Transporting information and energy simultaneously," in Proc. IEEE ISIT'2008, Toronto, ON, CANADA, July 2008, pp. 1612 1616.

[3] X. Zhou, R. Zhang, and C. K. Ho, "Wireless information and power transfer: Architecture design and rate-energy tradeoff," IEEE Trans. Commun., vol. 61, no. 11, pp. 4754 - 4767, Nov. 2013.

[4] A. Nasir, Z. Xiangyun, S. Durrani, and R. Kennedy, "Relaying protocols for wireless energy harvesting and information processing," IEEE Trans. Wireless Commun., vol. 12, no. 7, pp. 3622 - 3636, July 2013.

[5] A. Nasir, X. Zhou, S. Durrani, and R. Kennedy, "Wireless-powered relays in cooperative communications: Time-switching relaying protocols and throughput analysis," IEEE Trans. Commun., vol. 63, no. 5, pp. 1607 - 1622, May 2015.

[6] Z. Ding, S. Perlaza, I. Esnaola, and H. Poor, "Power allocation strategies in energy harvesting wireless cooperative networks," IEEE Trans. Wireless Commun., vol. 13, no. 2, pp. 846 - 860, Feb. 2014.

[7] S. Zhou, T. Chen, W. Chen, and Z. Niu, "Outage minimization for a fading wireless link with energy harvesting transmitter and receiver," IEEE J. Sel. Areas Commun., vol. 33, no. 3, pp. 496 - 511, Mar. 2015.

[8] C. Huang, R. Zhang, and S. Cui, "Optimal power allocation for outage probability minimization in fading channels with energy harvesting constraints," IEEE Trans. Wireless Commun., vol. 13, no. 2, pp. 1074 1087, Feb. 2014.

[9] H. Wang, W. Wang, and Z. Zhang, "Opportunistic wireless information and energy transfer for sustainable cooperative relaying," in $I C C$, London,UK, June 2015, pp. 160-165.

[10] Z. Zhou, M. Peng, Z. Zhao, and Y. Li, "Joint power splitting and antenna selection in energy harvesting relay channels," IEEE Signal Process. Lett., vol. 22, no. 7, pp. 823 - 827, July 2015.

[11] Y. Gu and S. Aissa, "RF-based energy harvesting in decode-and-forward relaying systems: Ergodic and outage capacities," IEEE Trans. Wireless Commun., vol. 14, no. 11, pp. 6425 - 6434, Nov. 2015.

[12] D. EU FP7 INFSO-ICT-247733 EARTH, "Energy efficiency analysis of the reference systems, areas of improvements and target breakdown," Jan. 2012.

[13] J. Li, E. Bjornson, T. Svensson, T. Eriksson, and M. Debbah, "Joint precoding and load balancing optimization for energy-efficient heterogeneous networks," IEEE Trans. Wireless Commun., vol. 14, no. 10, pp. $5810-5822$, Oct. 2015.

[14] R. M. Corless, G. H. Gonnet, D. E. G. Hare, D. J. Jeffrey, and D. E. Knuth, "On the Lambert W function," Advances in Computational Mathematics, vol. 5, pp. 329-359, 1996.

[15] I. S. Gradshteyn and I. M. Ryzhik, Table of Integrals, Series and Products. Academic Press, 2000. 